\title{
Neural Response to Emotional Salience in Schizophrenia
}

\author{
Stephan F Taylor*,', K Luan Phan ${ }^{2}$, Jennifer C Britton ${ }^{3}$ and Israel Liberzon ${ }^{1,4}$ \\ 'Department of Psychiatry, University of Michigan, Ann Arbor, MI, USA; ${ }^{2}$ Department of Psychiatry, University of Chicago, IL, USA; ${ }^{3}$ Neuroscience \\ Program, University of Michigan, Ann Arbor, MI, USA; ${ }^{4}$ Department of Psychiatry, Veterans Administration Medical Center, Ann Arbor, MI, USA
}

\begin{abstract}
Neuroimaging probes of brain regions implicated in emotion represent an important research strategy for understanding emotional dysfunction in schizophrenia. Anterior limbic structures, such as the ventral striatum and the amygdala, have been implicated in the pathophysiology of schizophrenia and the generation of emotional responses, although few studies to date have used emotion probes to target these areas in schizophrenia. With this goal in mind, emotionally salient visual images were used in a simple, nondemanding task. In all, 13 medicated, schizophrenic patients, five unmedicated patients, and 10 healthy volunteers viewed complex visual pictures and a nonsalient, blank screen while regional cerebral blood flow was measured with the [O-15] water technique. Pictures consisted of real world scenes with aversive, positive, and nonaversive content. Eye movements were recorded simultaneous with scan acquisition. Positron emission tomography images were analyzed for baseline, tonic activity, in addition to phasic changes ('activation') to salient stimuli. Lateral eye movement measures and on-line ratings showed good behavioral compliance with the task. Patients with schizophrenia showed impaired neural responses to salient stimuli in the right ventral striatum (VS), and they exhibited elevated tonic activity levels in the right VS and bilateral amygdala, inversely correlated with overall symptom severity. The patients also showed reduced modulation of visual cortex by salient stimuli. The results show that patients with schizophrenia exhibit impaired neural responses to emotionally salient stimuli in the VS, supporting a role for this structure in the pathophysiology of the illness. Reduced modulation of visual cortex by emotionally salient stimuli also suggests a failure to organize cerebral activity at a global level.

Neuropsychopharmacology (2005) 30, 984-995, advance online publication, 2 February 2005; doi: I 0. I038/sj.npp. I300679
\end{abstract}

Keywords: schizophrenia; amygdala; emotion; emission-computed tomography; visual cortex; psychophysiology

\section{INTRODUCTION}

Schizophrenia clearly impairs emotional functioning in patients, who show both reduced emotional expression, in the form of negative symptoms (Andreasen, 1982; Carpenter et al, 1988), as well as excessive emotions, such as those associated with persecutory delusions. The mechanisms of emotional disturbance in schizophrenia remain poorly understood, but an emerging functional anatomy of emotion suggests experimental leverage that might reveal some of the large-scale circuitry behind the symptoms. Several regions of the brain appear to mediate responses to salient stimuli that lead to appetitive and aversive behaviors, areas implicated in emotion. Anterior, limbic/ paralimbic regions, including the amygdala (Davis, 1992; Ledoux, 1992; Adolphs et al, 1995), ventral striatum (Nauta and Domesick, 1982; Haber and McFarland, 1999), anterior

*Correspondence: Dr SF Taylor, Department of Psychiatry, University of Michigan Medical Center, UH 9D Box 0I I8, I500 E Medical Center Drive, Ann Arbor, MI 48 109-0 I 18, USA, Tel: + I 734936 9760, Fax: + | 734936 7868, E-mail: sftaylor@umich.edu

Received 12 August 2004; revised 12 November 2004; accepted 6 December 2004

Online publication: 2 I December 2004 at http://www.acnp.org/citations/ NPPI $22104040364 /$ default.pdf cingulate cortex (Devinsky et al, 1995), insula (Augustine, 1985), and orbitofrontal cortex (Rolls, 1996) play central roles in emotional behavior, in addition to receiving extensive mesocortical and mesolimbic dopaminergic innervation (Joyce et al, 1991; Haber and Fudge, 1997; Meador-Woodruff et al, 1997). Given the role of dopamine in the pathophysiology of schizophrenia (Seeman, 1987; Davis et al, 1991; Grace et al, 1998), probes directed at these limbic/paralimbic regions are particularly relevant. Emotionally salient stimuli are also processed in regions outside limbic/paralimbic brain, such as the medial prefrontal cortex, thought to be critical for mediating interactions between cognition and emotion (Gusnard et al, 2001; Ochsner et al, 2002; Taylor et al, 2003). Neuroimaging work has also demonstrated that emotionally salient visual stimuli lead to increased activity in visual cortex (Phan et al, 2002), which may reflect top-down processing to enhance processing of salient stimuli (Pessoa et al, 2002). Taken together, this emerging neuroanatomy presents a reasonable framework in which to interpret emotional dysfunction in schizophrenia.

Several recent studies have used emotionally salient stimuli to probe emotions and relevant circuitry in schizophrenia. Using an odor challenge, Crespo-Facorro et al (2001) found that unmedicated schizophrenic patients 
failed to activate limbic and paralimbic cortex (insular cortex, nucleus accumbens, and parahippocampal gyrus). In medicated schizophrenic patients, the left amygdala and bilateral hippocampus failed to activate while passively viewing faces (Gur et al, 2002), both amygdalae failed to respond during sadness induction while viewing moodcongruent faces (Schneider et al, 1998), and the right amygdala failed to activate to nonaversive, salient visual content (Taylor et al, 2002; Takahashi et al, 2004). Both medicated (Phillips et al, 1999) and unmedicated schizophrenic patients (Paradiso et al, 2003) have demonstrated a failure to organize regional cerebral blood flow while viewing emotional pictures (Paradiso et al, 2003) or emotional faces (Phillips et al, 1999; Williams et al, 2004). More specifically, patients did not exhibit the normal modulation of visual cortical processing to emotionally salient stimuli (Taylor et al, 2002; Paradiso et al, 2003; Takahashi et al, 2004; Williams et al, 2004).

Work to date suggests that patients with schizophrenia do not activate regions important for the determination of emotional salience, but several different mechanisms might account for impaired activation. Neural responses to a behavioral challenge - that is, 'phasic' activity - say nothing about sustained, baseline activity, that is, 'tonic' activity. For example, we found less phasic change to salient stimuli in schizophrenic patients, relative to healthy controls, whereas we noted nonsignificantly greater tonic activity in the amygdala while viewing salient and nonsalient stimuli, raising the possibility that patients had tonic elevation of amygdaloid activity (Taylor et al, 2002). Elevated tonic activity could represent nonspecific activation of threatrelated neural circuitry (Davis, 1992; Ledoux, 1992; Adolphs et $a l, 1995)$ and a consequent failure to respond to an emotional challenge. Studies that have probed amygdala function in schizophrenia have measured changes in blood oxygenation level (Schneider et al, 1998; Phillips et al, 1999; Gur et al, 2002; Takahashi et al, 2004) or only reported changes in cerebral perfusion with a task (Crespo-Facorro et al, 2001; Paradiso et al, 2003). These studies have either not reported or not measured tonic activity.

The current study employed a neurobehavioral probe with emotional and nonemotional stimuli to assess both tonic and phasic neural activity with positron emission tomography (PET). The PET technique, with [O-15] water as a radiotracer, permitted the measurement of relative cerebral blood flow during each condition (tonic activity) and changing with the salience of stimuli containing aversive and positive emotional content (phasic activity). The use of positive stimuli was an important addition over our prior study (Taylor et al, 2002), since patients with schizophrenia tend to report equal intensity of negative experience as controls, but reduced intensity of positive experience (Myin-Germeys et al, 2000). The prediction was tested that schizophrenic patients would exhibit less phasic activity (activation to salient stimuli) and greater tonic activity in the amygdala and ventral striatum (VS). Although emotional tasks typically put minimal demands on subjects, it is possible that some of the findings, such as reduced modulation of visual cortex (Taylor et al, 2002; Paradiso et al, 2003; Takahashi et al, 2004; Williams et al, 2004), might reflect reduced eye movements while viewing the salient stimuli. In an attempt to rule out this possibility, we also measured eye movements while our subjects viewed the salient visual stimuli.

\section{METHODS}

\section{Subjects}

From a university-staffed community mental health center, 15 stable, medicated outpatients entered the study. Five, recently hospitalized, unmedicated patients were also recruited (two naïve to antipsychotic therapy, three off antipsychotic treatment $>6$ months). All patients were free of significant medical or neurological illness. A diagnosis of schizophrenia according to DSM-IV criteria (American Psychiatric Association, 1994) was established by a Structured Clinical Interview for Diagnosis (First et al, 1996). One of the unmedicated patients only met criteria for psychosis NOS at the time of the scan; however, 6-month follow-up revealed the persistence of psychosis, hence her diagnosis was changed to schizophrenia.

A total of 10 healthy control subjects were recruited from community advertisements, selected from the same age range as the patients. They were not taking medication, without any Axis I psychiatric disorders (Structured Clinical Interview for Diagnosis, non-patient version (First et al, 1996)) and without any psychosis in first-degree relatives.

The purpose and risks of the study were explained to all subjects, who gave written, informed consent to participate, as approved by the local institutional review board. The results from the healthy controls have been previously reported (Liberzon et al, 2003).

\section{Task Design}

The task consisted of viewing pictures with salient emotional content, obtained from the International Affective Picture System (IAPS; Lang and Greenwald, 1988).

Three sets of 18 images each were selected from the IAPS, plus supplements (approximately $10 \%$ of all images), consisting of positive (POS) pictures (animals, children, food), aversive (AV) pictures (mutilation, dead bodies), and nonaversive (NA) pictures (faces with neutral expressions, benign scenes). A fourth condition (blank condition-BL) consisted of a white fixation cross on a gray background (five different shades). The sets (POS, AV, NA) were transformed into black and white, luminance was balanced across the four sets (Photoshop 4.0, Adobe Systems), and they were matched with respect to faces and human figures.

\section{Eye Movement Recording}

Eye movement data were recorded using an MP-100 psychophysiological monitoring system (BioPac Systems, Santa Barbara, CA) to record horizontal electro-oculograms (EOG). The percentage of time lateral gaze fell outside the bounds of the monitor screen was calculated. Total scanning of the image was expressed as the standard deviation of eye position, which was log transformed for analysis in a mixed-model, two-way ANOVA, with task condition as a within-subjects factor and subject type as the between-subjects factor. 


\section{PET Image Acquisition}

During the scanning sessions, subjects viewed nine blocks of images. Images were displayed consecutively ( $5 \mathrm{~s}$ each) on a computer monitor suspended $40 \mathrm{~cm}$ from the subject, using SuperLab (Cedrus, Inc.). The first block consisted of neutral stimuli without acquisition of PET data, allowing subjects to adjust to the scanner environment. After this initial block, scan acquisition began. Eight scans were acquired, in two identically ordered sets of four blocks from each of the four conditions (BL, NA, AV, POS), with order counterbalanced in a partial Latin square. Subjects were instructed to focus on feelings that they experienced while watching the pictures. During stimulus presentation, subjects rated images according to a 5-point scale (from 1, 'very pleasant' to 5, 'very unpleasant'). For each blank screen, the subjects said ' 3 '. Subjects practiced the task prior to scanning to ensure their comprehension and performance.

A Siemens CTI 931-08/12 PET scanner (CTI Inc, Knoxville, TN) acquired 15 axial slices over an axial length of $10 \mathrm{~cm}$, capturing inferior, limbic structures and excluding the superior parietal lobe. A transmission scan measured by external ${ }^{68} \mathrm{Ge} /{ }^{68} \mathrm{Ga}$ ring sources preceded emission scanning. A small dose $(8 \mathrm{mCi})$ of radiotracer, injected prior to actual data acquisition but while the subjects viewed the initial block of neutral pictures, revealed the bolus transit time to the brain, in order to time stimulus presentation. For each emission scan, an i.v. bolus injection of $50 \mathrm{mCi}$ of $\left[{ }^{15} \mathrm{O}\right] \mathrm{H}_{2} \mathrm{O}$ was administered, and data collection began $5 \mathrm{~s}$ after arrival of radioactivity in the brain, continuing for $60 \mathrm{~s}$. Eight PET scans, each separated by $10 \mathrm{~min}$, were acquired for each subject. Stimuli presentation began $15 \mathrm{~s}$ prior to image acquisition, and continued for the first $30 \mathrm{~s}$ of each scan (uptake phase of the tracer), so as to maximize changes in the PET signals associated with the task of interest (Cherry et al, 1993). The second $30 \mathrm{~s}$ of each scan always contained blank stimuli with the fixation cross, exactly as in the 'blank' condition.

\section{PET Data Analysis}

Analysis of the PET data first employed a standardization process for each image which enabled averaging of image data both within and across subjects. Two sets of analyses occurred: an a priori volume of interest (VOI) analysis, which enabled the analysis of tonic activity, and a voxel-byvoxel, whole brain analysis, which enabled a more sensitive assay of phasic changes.

For the VOI analysis, a high-resolution normalization routine, developed in our laboratory, was applied to the data. Images were proportionally normalized, aligned within subjects, warped by a nonlinear transformation to standardize individual anatomy (Minoshima et al, 1993, 1994), and resliced into isotropic $2.25 \mathrm{~mm}$ voxels. From atlas coordinates (ICBM152 of the Montreal Neurological Institute), spherical volumes ( $15.75 \mathrm{~mm}$ diameter) of interest were placed over the right and left amygdala $(x= \pm 24 \mathrm{~mm} ; y=-3 \mathrm{~mm} ; z=-19 \mathrm{~mm})$. A VS VOI was constructed from two, contiguous, spherical volumes (13.5 mm diameter; $x, y, z= \pm 10,9,-8 ; \pm 15,-1,-8)$, including both the VS and the associated SLEA (Alheid and Heimer 1988). Activity was extracted from the anatomically standardized, individual images and analyzed in a mixedmodel, three-way ANOVA, with task condition (BL, NA, AV, POS) and side as within-subject factors and subject type (medicated patient, unmedicated patient, comparison subjects) as the between-subjects factor (Systat, Inc, Evanston, Il). Main effects of side and subject type constituted the analysis of tonic CBF, whereas the main effect of task condition represented phasic effects. Only subjects with a complete data set, that is, at least one observation for each condition, were entered into this analysis.

The whole brain, voxel-by-voxel analysis utilized Statistical Parametric Mapping (SPM99, Welcome Institute of Cognitive Neurology) to assess phasic changes occurring between the task conditions. Reconstructed images were realigned to the first volume acquired for each subject, anatomically normalized, resliced into $2 \times 2 \times 2 \mathrm{~mm}$ voxels, adjusted for global values (ANCOVA by subject) and smoothed (12 mm FWHM in three dimensions). A single, general linear model, modeling subject by condition effects, fitted each group. Subject-specific effects for each condition were then entered into a second-level, random effects analysis for three contrasts of interest: NA-BL; AV-NA; POS-NA. Voxel-by-voxel maps of phasic differences were obtained and thresholded for $Z$-scores at an uncorrected probability of $p<0.01$, which yielded ' $k$,' the size in voxels of a contiguous cluster of activation. Activation foci were examined when the peak magnitude of activation exceeded a $Z$-score with an uncorrected probability of $p<0.001$. Group differences were examined with voxel-by-voxel, paired $t$ tests for all positive activation foci from each contrast.

\section{RESULTS}

\section{Behavioral Results}

All subjects tolerated exposure to the images without significant difficulty. In debriefing interviews, no patients reported any exacerbation of symptoms while viewing the pictures. One medicated, schizophrenic subject had excessive $(>1 \mathrm{~cm})$, gross head movement, and a technical failure corrupted the scan data of another medicated schizophrenic patient. Both of these subjects were excluded from analysis. Of the remaining subjects, two scans for two medicated patients and one scan for one control subject were not obtained due to technical failures. One unmedicated, schizophrenic subject requested a bathroom break, leaving two $\mathrm{BL}$, one $\mathrm{NA}$, and one $\mathrm{AV}$ scans for analysis. Thus, for the final analysis, we had 13 medicated schizophrenic patients, five unmedicated schizophrenic patients (one patient with an incomplete data set), and 10 control subjects (Table 1). Image data was not available for two control subjects at the level of the amygdala, and one subject at the level of VS, where the brain fell outside the field of view of the scanner.

On-line ratings of stimuli showed clear effects of the intended manipulation of valence (Table 2). Comparisons between groups showed that the patients rated the POS stimuli as less positive than the control subjects. There were no significant differences between groups for the NA or the AV stimuli. There were no significant differences between the medicated and unmedicated patients in their ratings of the salient pictures. 
Table I Demographic and Clinical Characteristics of Subjects

\begin{tabular}{|c|c|c|c|}
\hline Mean \pm SD & $\begin{array}{c}\text { Medicated } \\
\text { schizophrenic } \\
\text { patients } \\
(n=13)\end{array}$ & $\begin{array}{c}\text { Unmedicated } \\
\text { schizophrenic } \\
\text { patients } \\
(n=5)\end{array}$ & $\begin{array}{l}\text { Healthy } \\
\text { control } \\
\text { subjects } \\
(n=10)\end{array}$ \\
\hline Age, years & $35.2 \pm 11.9$ & $25.4 \pm 8.0$ & $27.5 \pm 8.6$ \\
\hline (range) & $20-54$ & $18-38$ & $20-46$ \\
\hline Males & 9 & 2 & 6 \\
\hline Socioeconomic status ${ }^{\mathrm{a}}$ & $2.9 \pm 0.5$ & $3.2 \pm 0.8$ & $2.6 \pm 0.5$ \\
\hline $\begin{array}{l}\text { Duration ill, years } \\
\text { (range) }\end{array}$ & $\begin{array}{c}16.3 \pm 11.0 \\
\mid-34\end{array}$ & $\begin{array}{c}5.6 \pm 7.6 \\
1-19\end{array}$ & - \\
\hline Age of onset, years & $18.9 \pm 3.8$ & $19.8 \pm 3.3$ & - \\
\hline (range) & $10-24$ & $17-25$ & \\
\hline \multicolumn{4}{|l|}{ Medications } \\
\hline Clozapine & 8 & & \\
\hline Mean dose & $375 \pm 128 \mathrm{mg}$ & & \\
\hline Other atypicals & 5 & & \\
\hline $\begin{array}{l}\text { Mean dose } \\
\text { (risperidone } \\
\text { equivalent) }\end{array}$ & $5.6 \pm 2.6 \mathrm{mg}$ & & \\
\hline \multicolumn{4}{|l|}{ Symptom ratings } \\
\hline BPRS $^{\mathrm{b}}$ total & $33.6 \pm 8.7$ & $45.4 \pm 5.4$ & \\
\hline BPRS positive & $9.9 \pm 4.1$ & $15.0 \pm 1.6$ & \\
\hline BPRS negative & $8.1 \pm 3.5$ & $7.0 \pm 2.9$ & \\
\hline SANS $^{C}$ global sum & $8.4 \pm 4.7$ & $6.4 \pm 3.1$ & \\
\hline
\end{tabular}

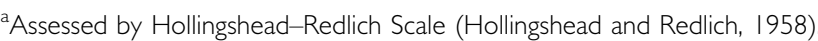

bBrief Psychiatric Rating Scale (Overall and Gorham, 1962).

'Schedule for the Assessment of Negative Symptoms (Andreasen, 1983).

Table 2 Ratings of Visual Stimuli

\begin{tabular}{|c|c|c|c|c|}
\hline Condition $^{\mathrm{a}}$ & $\begin{array}{c}\text { Schizophrenic } \\
\text { patients }\end{array}$ & $\begin{array}{c}\text { Healthy } \\
\text { control } \\
\text { subjects }\end{array}$ & t-value & $p$ \\
\hline Positive pictures (POS) & $1.75 \pm 0.27$ & $1.46 \pm 0.28$ & 2.69 & 0.01 \\
\hline Nonaversive pictures (NA) & $2.57 \pm 0.24$ & $2.67 \pm 0.22$ & 0.54 & 0.59 \\
\hline Aversive pictures (AV) & $4.64 \pm 0.12$ & $4.69 \pm 0.06$ & 0.42 & 0.68 \\
\hline
\end{tabular}

${ }^{a}$ Ratings on a 5 -point scale, from I (pleasant) to 5 (unpleasant), \pm SD.

Effect of condition: $F[2,52]=443, p<0.0001$.

$\mathrm{NA}>\mathrm{POS}, t[27]=9.3, p<0.000 \mathrm{I}$

$\mathrm{AV}>\mathrm{NA}, t[27]=|7.5, p<0.000|$

$\mathrm{AV}>\mathrm{POS},[27]=32.1, p<0.0001$

\section{Eye Movements}

The EOG data revealed that all analyzed subjects looked at the images and maintained lateral eye position within the bounds of the display screen for $100 \%$ of the scans for the control subjects, and $96 \%$ of the scans for the patients. Four patients showed eye positions away from the display screen in the following amounts: one medicated patient(POS- $0.5 \%$ ), one unmedicated patient (NA- $0.4 \%$; POS-3\%), one unmedicated patient (POS-0.5\%), one unmedicated

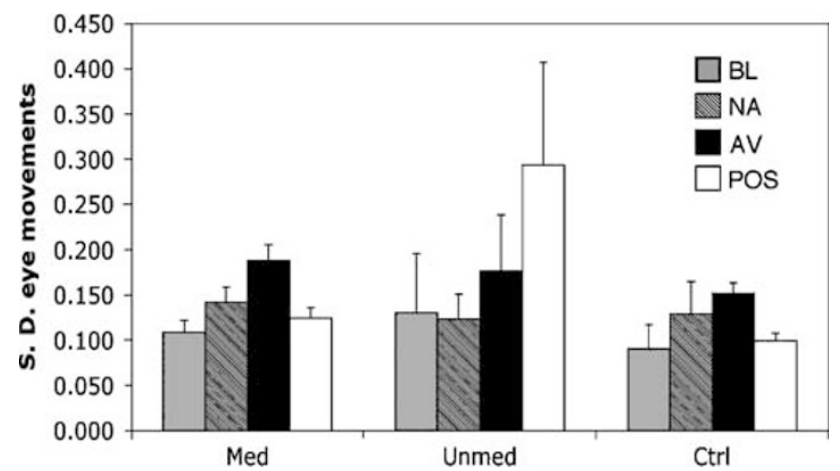

Figure I Lateral eye movements for medicated (Med) schizophrenic patients, unmedicated schizophrenic patients (Unmed), and control subjects (Ctrl) while viewing pictures. BL: blank condition; NA: nonaversive condition; AV: aversive condition; POS: positive condition. Units are in standard deviation (SD) of lateral eye movements.

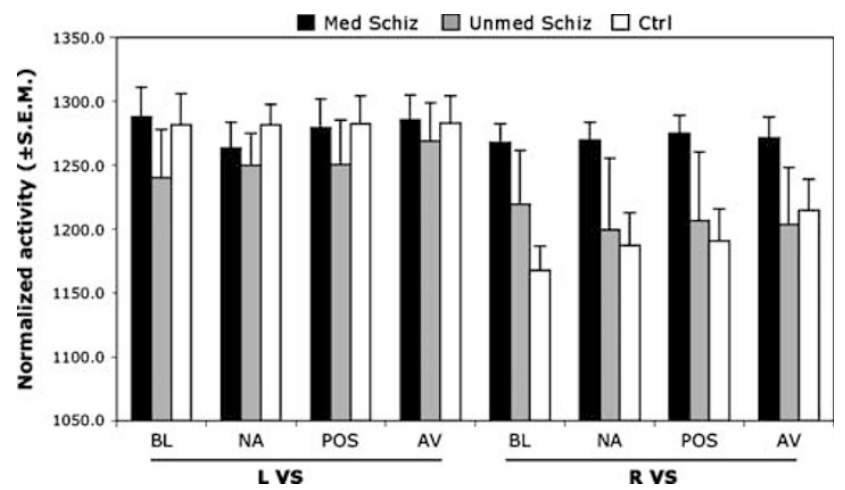

Figure 2 Normalized, tonic activity (arbitrary units) in the a priori regions placed on the right and left ventral striatum (VS). There was a significant side-by-subject type interaction (see text for statistical details). Abbreviations as in Figure I.

patient (BL-3.6\%; AV-4.3\%). ANOVA of the log-transformed eye movements showed a significant main effect of condition $(\mathrm{F}[3,72]=13.3, p<0.0001)$. Figure 1 demonstrates an identical pattern of viewing across the conditions for the medicated patients and the control subjects, in the following order: $\mathrm{AV}>\mathrm{NA}>\mathrm{POS}>\mathrm{BL}$. There were no significant effects of group $(\mathrm{F}[2,24]=0.6, p=0.56)$, but a trend towards a significant condition-by-group interaction was noted $(\mathrm{F}[6,72]=1.85, p=0.11)$, which appeared as slightly greater eye movements in the POS condition for the unmedicated patients.

\section{Volume of Interest Analysis in the VS and Amygdala}

Overall, CBF in the VS exhibited greater tonic activity on the left side (See Figure 2; effect of side: F[2, 23] =17.16, $p=0.000$ ), and there was a significant interaction between subject type and side $(\mathrm{F}[2,23]=6.72, p=0.005)$. As Figure 2 shows, the medicated patients had greater tonic activity in the right VS than the control subjects, and little sign of leftlaterality. The overall effect of subject type was not significant for tonic activity $(\mathrm{F}[2,23]=1.59, p=0.22)$. There were no other significant main effects or interactions $(p>0.20)$. 
In the amygdala, the patients showed a trend towards greater tonic activity on both sides (effect of subject type $\mathrm{F}[2,22]=3.13, p=0.06$; Figure 3 ). As in the VS, tonic activity on the left was greater than the right $(\mathrm{F}[1,22]=4.36$, $p=0.05$ ), but unlike the VS, all three groups exhibited leftlaterality in the amygdala. For phasic changes to condition, the polynomial test of order was calculated, with conditions arranged in the following sequence: BL, NA, POS, AV. This order reflected the fact that activation of the amygdala occurs with positive stimuli, but is more likely to occur with aversive stimuli (Phan et al, 2002). We found a significant linear effect for condition $(\mathrm{F}[1,22]=10.05, p=0.004)$. Post hoc tests revealed significant pairwise comparisons in the left amygdala for AV $>\mathrm{BL}(t[24]=2.13, p=0.04)$ and in the right amygdala for $\mathrm{AV}>\mathrm{BL}(t[24]=2.28, p=0.03)$, $\mathrm{AV}>\mathrm{NA}(t[24]=2.73, p=0.01)$, and $\mathrm{AV}>\mathrm{POS}(t[24]=$ $2.47, p=0.02)$. There were no significant interactions with subject type for any of these main effects or first-order linear effects $(p>0.4)$.

Direct comparison of the unmedicated and medicated patients revealed no significant group differences for this VOI analysis in the VS and amygdala $(p>0.20)$, although the small number of unmedicated subjects limits the power of this analysis. Combining the two patient groups yielded essentially the same results, except that the trend for greater tonic activity in the amygdala among the patients became significant (main effect of subject type, $\mathrm{F}[1,23]=6.45$, $p=0.02)$.



Figure 3 Normalized, tonic activity (arbitrary units) in the a priori regions placed on the right and left amygdala. There was a significant effect of side, and a trend effect for subject type (see text for statistical details). Abbreviations as in Figure 2.

Table 3 Correlations between Tonic Activity in a Priori Volumes of Interest and Symptoms

\begin{tabular}{lllll}
\hline Symptoms & L VS & R VS & L amygdala & R amygdala \\
\hline SANS & $-0.52 *$ & -0.12 & -0.5 ।* & -0.06 \\
BPRS positive symptoms & $-0.47 *$ & $-0.49 *$ & -0.34 & -0.24 \\
BPRS total symptoms & $-0.55^{*} *$ & $-0.57 * *$ & $-0.53 *$ & -0.31 \\
\hline * $p<0.05$. & & & & \\
$* * * 0.02$. & & & & \\
\hline
\end{tabular}

Neuropsychopharmacology

\section{Volume of Interest Analysis: Correlation with Symptoms}

Calculation of the Pearson correlation coefficients revealed that greater symptom severity (positive, negative, and total BPRS ratings) correlated with less tonic activity in the bilateral VS and the left amygdala, averaged over all four conditions (see Table 3). This pattern was essentially unchanged when we looked at the correlation of each condition with symptoms, separately. Examination of scattergrams for the bivariate correlations did not show any pattern attributable to medicated/unmedicated status or particular medication, for example, clozapine $v s$ other atypical antipsychotics. We also looked for correlations between tonic and phasic activity, and found none significant.

\section{Voxel-by-Voxel Analysis of Phasic Activity in the VS and Amygdala}

In the voxel-by-voxel analysis, the controls activated the right VS for POS-NA, and this activation was significantly greater compared with the patients $(10,20,-2, Z=3.54$, $k=173)$. In the contrast of $\mathrm{AV}-\mathrm{NA}$, control subjects activated the right VS (at a trend level, $Z=3.07$ ). In the group comparison, the control subjects exhibited more VS phasic activity than the patients $(14,14,-4, Z=3.14$, $k=240$ ).

For the control subjects, a cluster of activation for the NA-BL contrast occurred in the left medial temporal region, which included the amygdala (Table 4). However, the group comparison did not reveal significant differential activation (controls $>$ patients). There were no other activations in the amygdala for the other contrasts, for either group.

\section{Voxel-by-Voxel Analysis of Phasic Activity in Visual Cortex}

Activation for NA relative to $\mathrm{BL}$ gave abundant $\mathrm{CBF}$ changes in the posterior cortex, including visual processing regions of the occipital cortex, and downstream areas such as the fusiform gyrus. The patients exhibited nominally higher magnitude and extent of activation in the visual cortex $(12 \%$ more voxels activated). However, in the direct comparison of the groups, controls exhibited higher activity in the right lingual gyrus $(10,-44,-2, Z=3.29, k=180 ; 26,-70,2$, $Z=3.19, k=118)$. There were no foci in the visual cortex where phasic activity was greater for patients.

SPM analysis showed robust phasic modulation of visual cortical regions for AV-NA in both groups (see Table 5 and Figure 4a). Controls exhibited greater magnitude and extent of activation than patients, and this was significantly greater in the left lingual gyrus $(-18,-52,10, Z=4.32, k=347)$, and the left lateral occipital gyrus $(-26,-72,16, Z=3.29$, $k=207$ ). For the POS-NA contrast, significant phasic modulation of visual cortical regions was also observed, for both groups (Table 6 and Figure 4b). As with AV-NA, the controls modulated visual cortex in response to the salient positive stimuli with greater magnitude and greater extent, significantly greater in the right superior occipital cortex $(24,-80,28, Z=3.55, k=173)$. There were no foci where patients exhibited greater activation than control subjects. 
Table 4 Activation Peaks, Nonaversive Minus Blanks

\begin{tabular}{|c|c|c|c|c|c|c|}
\hline \multirow[b]{2}{*}{ Region } & \multicolumn{3}{|c|}{ Healthy controls } & \multicolumn{3}{|c|}{ Schizophrenic patients } \\
\hline & $(x, y, z)^{a}$ & Cluster size $^{b}$ & Z-score & $(x, y, z)^{a}$ & Cluster size $^{b}$ & Z-score \\
\hline \multirow{2}{*}{ Occipital pole (17//8/19) } & $-24,-84,-8$ & & 5.05 & $30,-90,16$ & & 6.58 \\
\hline & $14,-84,-6$ & & 5.07 & $-14,-86,-16$ & & 6.49 \\
\hline \multirow[t]{2}{*}{ L fusiform gyrus (19/37) } & $-42,-24,-26$ & 175 & 3.36 & & & \\
\hline & $-32,-38,-26$ & 178 & 3.28 & & & \\
\hline L temporal pole/inf amygdala (38) & $-24,10,-36$ & 210 & 3.89 & & & \\
\hline R inferior frontal gyrus (45) & & & & $62,28,0$ & 51 & 3.21 \\
\hline Superior frontal gyrus (6) & $0,18,60$ & 56 & 3.67 & & & \\
\hline \multirow[t]{3}{*}{ Dorsomedial prefrontal cortex $(9 / 10)$} & $-6,60,36$ & 769 & 3.63 & $-6,54,36$ & 338 & 3.49 \\
\hline & $0,56,42$ & & 3.99 & $-12,48,46$ & & 3.18 \\
\hline & $24,62,28$ & & 3.42 & & & \\
\hline
\end{tabular}

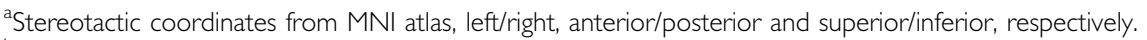

${ }^{b}$ Cluster size, number of voxels $(k)$.

\section{Voxel-by-Voxel Analysis of Phasic Activity in Other Brain Areas}

Tables 4-6 show all the regions of activation for each group, above the threshold of $p<0.001$. Both groups activated the dorsal medial prefrontal cortex (NA-BL), which has been reported in other studies with IAPS stimuli (Liberzon et al, 2003; Taylor et al, 2003). Comparison of activation between groups showed only a trend for greater activation by the control subjects $(10,48,50, Z=2.97, k=217)$; besides this trend, there were no other group differences.

\section{Voxel-by-Voxel Analysis: Effect of Medication}

The analyses described above were repeated, without the unmedicated patients. In general, the absence of the unmedicated patients did not change the results, with a few exceptions. In the contrast of NA-BL without the unmedicated patients, the control subjects showed significantly greater activation than medicated patients in a region inferior to and overlapping with the left amygdala $(-24,10$, $-36, Z=3.40, k=256)$. Also, with the removal of the unmedicated patients, the significant difference between the patients and controls for the right VS failed to reach significance for the AV-NA contrast. However, for the POSNA contrast, the group difference in the right VS retained significance for the medicated patients alone $(10,20,-2$, $Z=3.08, k=119)$. In general, the medicated patients alone yielded qualitatively similar patterns of activation as the medicated patients. The five unmedicated patients were compared to a matched subset of five control subjects. In spite of the low power of this comparison, the right VS still showed greater activation for the control subjects, but only for the POS-NA contrast $(10,16,-4, Z=3.55, k=197$, for 4 schizophrenic patients). Figure 5 shows differential phasic activation in the right VS between the control subjects and the unmedicated patients $(n=4)$ and a separate comparison with the 13 medicated patients.

\section{DISCUSSION}

This experiment used emotionally salient material to probe circuitry central to the pathophysiology of schizophrenia. As predicted, the patients showed smaller phasic changes to emotionally salient stimuli. They exhibited impaired phasic responses to salient stimuli in the right VS, and reduced phasic modulation of visual cortex by salient stimuli. Lateral eye movement measures and online ratings demonstrated good behavioral compliance with the task, arguing against gross performance differences explaining the group differences. In addition to the reduced phasic changes, we also found elevated tonic activity in the amygdala and right VS. Overall, the findings provide evidence that the neural response to emotionally salient stimuli is abnormal in schizophrenia. 
Table 5 Activation Peaks, Aversive Minus Nonaversive

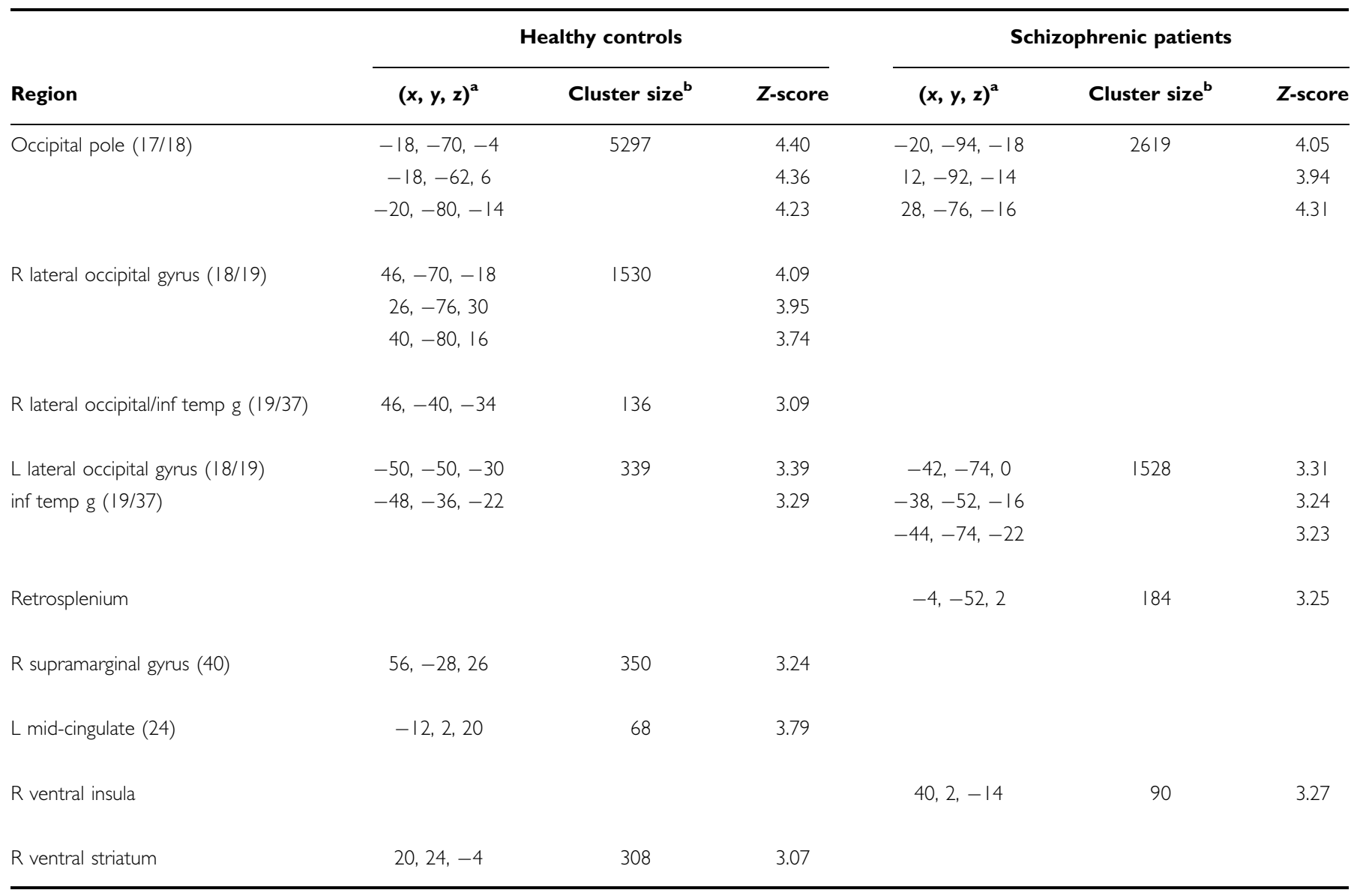

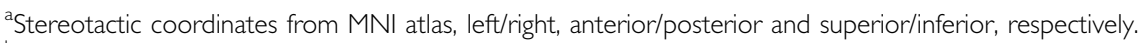

${ }^{b}$ Cluster size, number of voxels ( $k$ ).

\section{Response to Salient Stimuli in VS and Amygdala}

The brain has evolved systems that select salient stimuli, which signal potential dangers and rewards, and organize appropriate behavioral responses. Limbic neural circuits, such as the VS and amygdala, have key roles in the determination of responses to salient stimuli (Mogenson et al, 1980; Davis, 1992; Ledoux, 1992; Stern and Passingham, 1996; Parkinson et al, 2000). The fact that these circuits are also implicated in schizophrenic pathophysiology (Grace et al, 1998; Heimer, 2000) motivated this study. Phenomenologically, patients with schizophrenia do not respond appropriately to salient stimuli. They fail to express common emotions, clinically evident as negative symptoms, or they read unjustified meaning into stimuli, as in persecutory delusions. Experimentally, the electrophysiology literature shows that schizophrenic patients have impaired responses to salient stimuli, in the form of reduced P300 amplitudes in 'oddball' paradigms (Pfefferbaum et al, 1989; McCarley et al, 1991). However, this work has not targeted the VS and amygdala, and the P300 stimuli lack emotional content that would elicit appetitive or aversive processing.

The present study represents the first published work, of which are aware, demonstrating abnormal activation of the
VS in schizophrenia. The data parallel and extend prior work showing hypoactive phasic responses in other limbic structures (Schneider et al, 1998; Crespo-Facorro et al, 2001; Taylor et al, 2002; Paradiso et al, 2003). Both positive and aversive stimuli activated the VS in healthy subjects, a finding consistent with notions that this structure mediates a general response to emotionally salient stimuli with appetitive or aversive qualities (Berridge and Robinson, 1998; Horvitz, 2000; Liberzon et al, 2003). Significant differences between the control subjects with the medicated and unmedicated patients only occurred for the POS stimuli, although the combined group of patients exhibited impaired activation of the VS for both POS and AV stimuli. The stronger effect of POS stimuli may reflect the fact that patients with schizophrenia do not differ from healthy comparison subjects in their ratings of aversive stimuli in the laboratory (Kring et al, 1993; Kring and Neale, 1996; Earnst and Kring, 1999) and in the intensity of their aversive life experiences (Myin-Germeys et al, 2000). We did find that the patients gave less positive ratings of the positive pictures, consistent with reports of reduced positive life experiences (Myin-Germeys et al, 2000). In other words, the POS stimuli may have been better suited to eliciting group differences. 

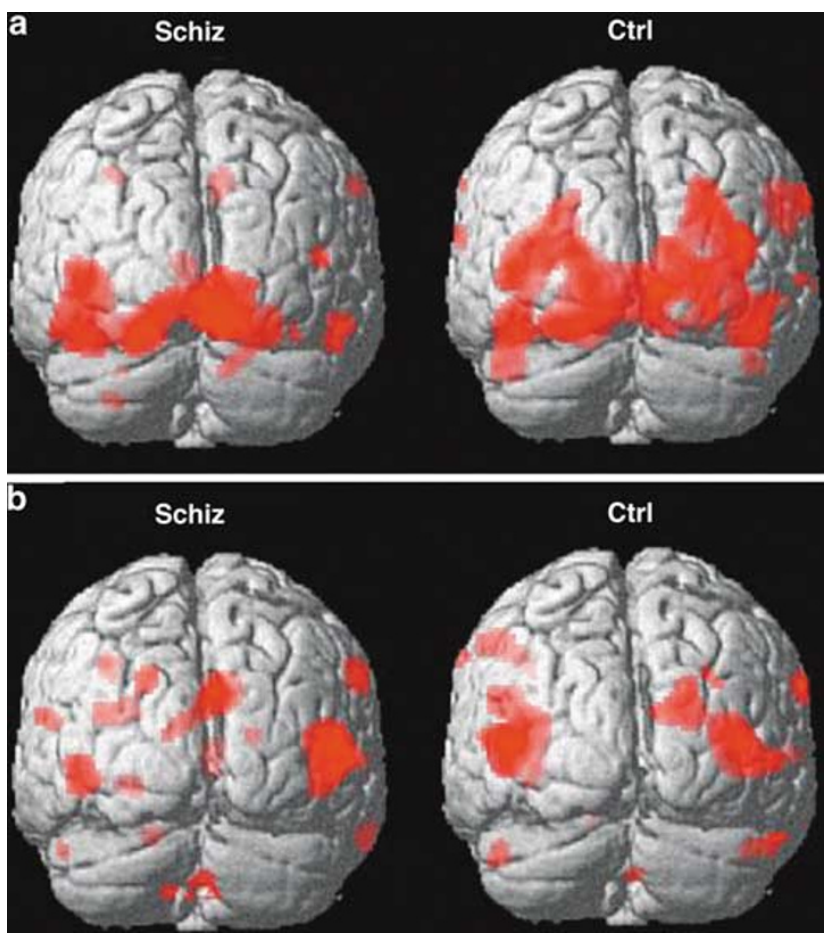

Figure 4 Phasic changes in posterior cortex in response to: (a) aversive or (b) positive pictures, compared to nonaversive pictures, for all schizophrenic (Schiz) and control (Ctrl) subjects. Activated voxels $(p<0.01$, uncorrected) are projected onto a surface rendering of a reference brain.

Our data did not support the hypothesis that tonic hyperactivity of the amygdala/VS would underlie impaired phasic responses, possibly related to symptom generation. Several investigators have suggested that the amygdala, or VS, produce the positive symptoms of psychosis through aberrant biasing of neural circuits to distort reality construction (Fudge et al, 1998; Moore et al, 1999; Grossberg, 2000; Kapur, 2003). A specific hypothesis, emanating from these theories and some of our previous work (Taylor et al, 2002), would predict that tonic hyperactivity in these limbic regions would directly correlate with positive symptoms. In this formulation, patients would perceive omnipresent threats, and salience-detection circuits would operate continuously, but not selectively. The schizophrenic patients did show greater tonic activity in the right VS (and bilateral amygdala), although the data showed greater positive (and negative) symptoms correlated with less tonic activity-opposite the prediction.

The finding of an inverse correlation between symptoms and activity in the VS/amygdala is useful because it informs us about what tonic hyperactivity is not. However, interpreting the inverse correlation between tonic activity and symptoms presents a challenge. None of the emotionally salient stimuli elicited any symptoms in the patient, and stimuli associated with symptoms could have elicited a very different pattern of tonic and phasic change in the patients. It could reflect greater anxiety in the patients who were less afflicted with positive and negative symptoms. We sought to find third factors, such as education level, age of onset, and medication dose, that might explain these relationships, and we found no significant correlations. Given the modest strength of these correlations, the small variation in symptom magnitude, and the relatively low severity of the patients in the sample, additional speculation about causality should await replication of the finding.

In the VOI analysis of the VS, tonic hyperactivity appeared as a lack of the functional asymmetry (left $>$ right) found in the healthy subjects. Structural studies of the medial temporal lobe in schizophrenia have also identified a lack of normal asymmetry (Bilder et al, 1994, 1999; Pearlson et al, 1997), although it is difficult to say whether or not the same process underlies these findings. The unmedicated patients showed a pattern of activity closer to that of the control subjects, and the findings were strengthened somewhat when we removed the unmedicated patients, although the small number of unmedicated patients makes conclusions about the role of medications difficult. Therapy with dopamine-blocking agents does elevate functional activity in the basal ganglia, of which the VS forms the most ventral aspect (Buchsbaum et al, 1992; Wolkin et al, 1996; Miller et al, 1997). However, a medication effect cannot easily explain how the patients had equivalent activity as control subjects on the left and greater activity on the right.

Several other points about the findings require comment. The VOI findings did not show the effect of POS stimuli, relative to NA stimuli, for the healthy subjects because the VOI was several millimeters caudal of the VS focus of activation in the SPM analysis. Our a priori placement of this VOI depended upon prior work that only utilized aversive stimuli (Taylor et al, 2000). Animal studies (Reynolds and Berridge 2002, 2003) and human neuroimaging studies have suggested that while the VS processes both aversive and appetitive stimuli, the latter may occur in more rostral regions of this structure (Liberzon et al, 2003). Hence, we only found activation to POS stimuli in the lessconstrained SPM analysis.

Unlike our previous report (Taylor et al, 2002), we did not find significant group differences for phasic activity in the amygdala. In the VOI analysis, the patients showed a similar pattern of activation as the control subjects, demonstrating that patients can activate the amygdala in response to emotionally salient stimuli, at least to some degree. In the voxel-by-voxel analysis, changes in the amygdala in the healthy comparison group were present, but not particularly robust, a fact that reduced our power to detect group differences. The amygdalae, as well as the VS, are small structures, making them subject to partial volume effects with this PET camera (effective resolution $12 \mathrm{~mm}$ FWHM). Reductions in amygdala volume have been reported in schizophrenia (Nelson et al, 1998; Wright et al, 2000), although not uniformly (Chance et al, 2002). Of course, increased tonic activity cannot be accounted for by a normal or reduced volume of the amygdala.

\section{Modulation of Visual Cortex}

The patients showed significantly less modulation in several regions associated with processing visual information, replicating previous work (Taylor et al, 2002; Paradiso et al, 2003). The modulation of visual cortex by emotional salience is well acknowledged (Phan et al, 2002), and the phenomenon may represent top-down modulation to 
Table 6 Activation Peaks, Positive Minus Nonaversive

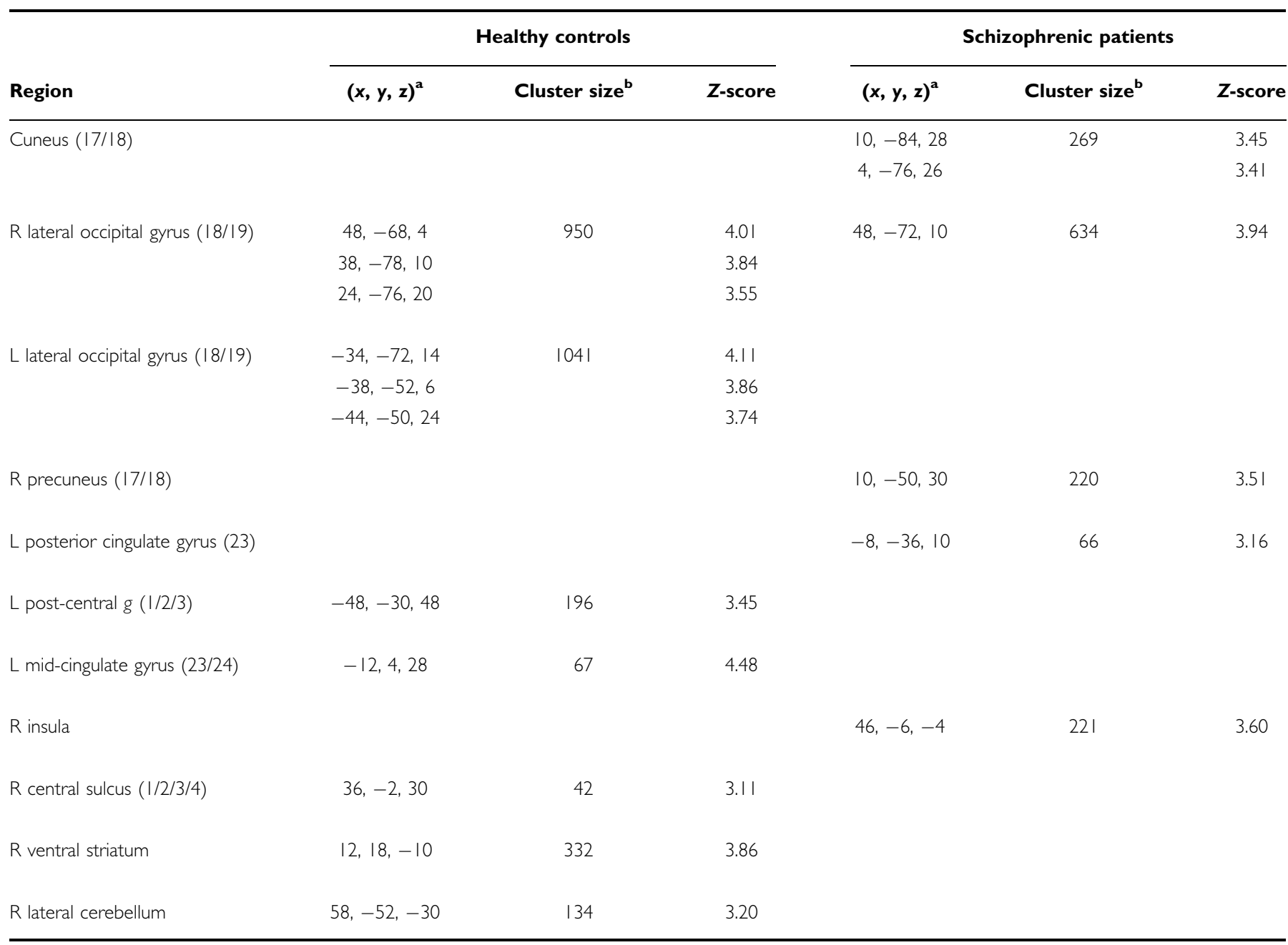

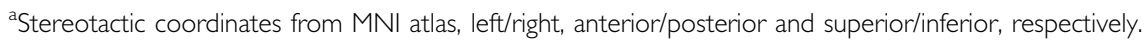

${ }^{\mathrm{b}}$ Cluster size, number of voxels $(k)$.
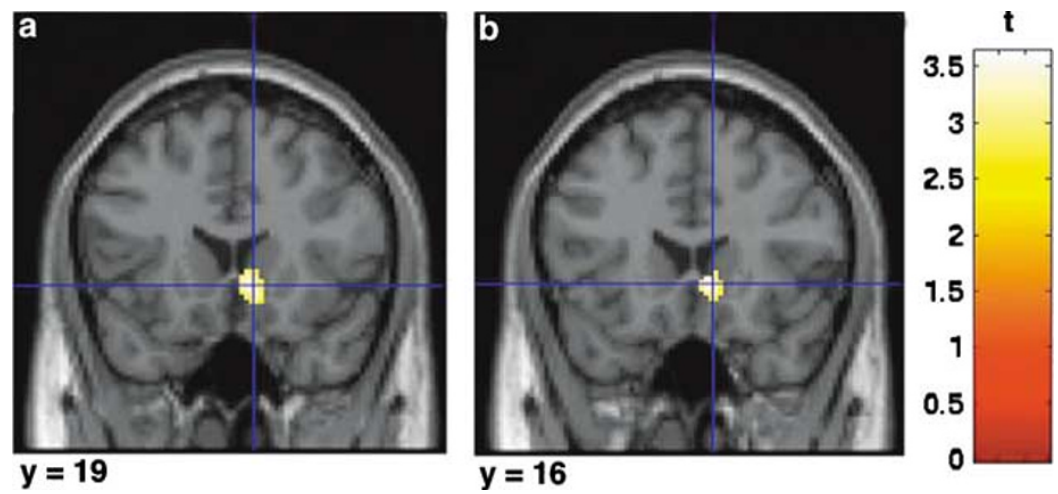

Figure 5 Phasic changes in the right ventral striatum for POS-NA, where control subjects show greater activation than patients. (a) Control subjects $(n=9)$ greater than medicated schizophrenic patients $(n=13)$; (b) Control subjects $(n=5)$ greater than unmedicated schizophrenic patients $(n=4)$. Voxels displayed at $p<0.01$.

enhance processing of emotional stimuli, that is, saliencemediated attention effects (Pessoa et al, 2002). Prior work in schizophrenia has not sufficiently measured eye movements, leaving unanswered the question of whether or not the patients scanned the pictures in a qualitatively different way, as other studies of visual scanning in schizophrenia have demonstrated (Phillips and David, 1994, 1998; Streit et al, 1997). The measurement of lateral EOG activity in the 
current study showed an identical pattern of eye movement magnitude across the conditions for the control and medicated schizophrenic subjects. This finding suggests that differences in visual scanning, which might have occurred, would not account for differences in modulation of visual cortex by emotional stimuli. We searched for correlations between EOG parameters and visual cortical modulation and found none. Even if the patients did scan the complex images differently, the similar magnitudes of eye movements between the four conditions suggest that they would scan pictures from the different conditions in the same way. Hence, a contrast of CBF between conditions should not yield phasic CBF activity due to differences in eye movements.

A smaller effect of emotionally salient content on visual cortical processing in schizophrenia may reflect a failure of a process, such as attention, interacting with emotionally salient content. Patients with schizophrenia have welldocumented impairments in attention (Nuechterlein and Dawson, 1984; Saykin et al, 1991). If modulation of visual cortex does represent top-down, attentional control, then reduced modulation of visual processing streams by salient content may reflect this attentional impairment. However, equating the strength of the phasic signal with attention may not capture the data accurately. The schizophrenic patients actually exhibited nominally larger magnitude and extent of activation in the visual cortex for the comparison of nonaversive stimuli with blanks. It has been often observed, but infrequently acknowledged, that patients with schizophrenia exhibit significantly greater posterior activity for simple visual stimuli (Siegel et al, 1993; Renshaw et al, 1994; Andreasen et al, 1997; Taylor et al, 1997). In spite of activating more voxels, the patients in the present study still exhibited significantly less phasic activity than controls in the lingual gyrus. One may interpret these results as evidence of a failure of an organizing process, similar to, but not identical with, attention. The ability to organize activity across regions of cortex has been suggested as one of the core deficits in schizophrenia (Andreasen et al, 1998), and the failure to modulate visual cortex to emotionally salient stimuli may reflect this poor organization.

\section{Conclusion}

This study used a neurobehavioral probe to target neural circuitry of hypothesized importance for schizophrenia, including brain regions with extensive dopaminergic innervation that process salient, emotional information. The data described in this experiment demonstrate that structures, such as the VS, show a blunted response to emotional salience. Furthermore, some of this same circuitry, along with the amygdala, may be tonically overactive. While many questions remain about the role of this 'salience circuitry' in producing the phenomena of schizophrenia, the use of salience probes can provide important experimental leverage.

\section{ACKNOWLEDGEMENTS}

We acknowledge the assistance of Laura R Decker and the technologists at the University of Michigan PET Center in the acquisition of data reported here. This work was supported by grants from the National Alliance for Research in Schizophrenia and Depression, the National Institute of Mental Health (K08 MH01258, R01 MH), Eli Lilly, and the University of Michigan General Clinical Research Center (M01 RR00042). This work has been presented in abstract form at the International Congress on Schizophrenia Research in Colorado Springs, Colorado, April 2003.

\section{REFERENCES}

Adolphs R, Tranel D, Damasio H, Damasio AR (1995). Fear and the human amygdala. J Neurosci 15: 5879-5891.

Alheid GF, Heimer L (1988). New perspectives in basal forebrain organization of special relevance for neuropsychiatric disorders: the striatopallidal, amygdaloid, and corticopetal components of substantia innominata. Neuroscience 27: 1-39.

Andreasen NC (1982). Negavtive vs positive schizophrenia. Arch Gen Psychiatry 39: 789-794.

Andreasen NC (1983). The Scale for the Assessment of Negative Symptoms. The University of Iowa: Iowa City.

Andreasen NC, O'Leary DS, Flaum M, Nopoulos P, Watkins GL, Boles Ponto LL et al (1997). Hypofrontality in schizophrenia: distributed dysfunctional circuits in neuroleptic-naive patients. Lancet 349: 1730-1734.

Andreasen NC, Paradiso S, O'Leary DS (1998). 'Cognitive dysmetria' as an integrative theory of schizophrenia: a dysfunction in corticalsubcortical-cerebellar circuitry? Schizophr Bull 24: 203-218.

Association AP (1994). Diagnostic and Statistical Manual of Mental Disorders, 4th edn. (DSM-IV). American Psychiatric Association: Washington, DC.

Augustine JR (1985). The insular lobe in primates including humans. Neurol Res 7: 2-10.

Berridge KC, Robinson TE (1998). What is the role of dopamine in reward: hedonic impact, reward learning, or incentive salience? Brain Res Brain Res Rev 28: 309-369.

Bilder RM, Wu H, Bogerts B, Degreef G, Ashtari M, Alvir JM et al (1994). Absence of regional hemispheric volume asymmetries in first-episode schizophrenia. Am J Psychiatry 151: 1437-1447.

Bilder RM, Wu H, Bogerts B, Robinson D, Ashtari M, Woerner M et al (1999). Cerebral volume asymmetries in schizophrenia and mood disorders: a quantitative magnetic resonance imaging study. Int J Psychophysiol 34: 197-205.

Buchsbaum MS, Potkin SG, Siegel BV, Lohr J, Katz M, Gottschalk LA et al (1992). Striatal metabolic rate and clinical response to neuroleptics in schizophrenia. Arch Gen Psychiatry 49: 966-974.

Carpenter WT, Heinreichs DW, Wagman AMI (1988). Deficit and non-deficit forms of schizophrenia: the concept. Am J Psychiatry 145: $578-583$.

Chance SA, Esiri MM, Crow TJ (2002). Amygdala volume in schizophrenia: post-mortem study and review of magnetic resonance imaging findings. Br J Psychiatry 180: 331-338.

Cherry SR, Woods RP, Mazziotta JC (1993). Improved signal-tonoise in activation studies by exploiting the kinetics of oxygen15-labeled water. J Cerebr Blood Flow Metab 13: S714.

Crespo-Facorro B, Paradiso S, Andreasen NC, O’Leary DS, Watkins GL, Ponto LL et al (2001). Neural mechanisms of anhedonia in schizophrenia: a PET study of response to unpleasant and pleasant odors. Jama 286: 427-435.

Davis KL, Kahn RS, Ko G, Davidson M (1991). Dopamine in schizophrenia: a review and reconceptualization. Am J Psychiatry 148: $1474-1486$.

Davis M (1992). The role of the amygdala in conditioned fear. In: Aggleton JP (ed). The Amygdala: Neurobiological Aspects of Emotion, Memory and Mental Dysfunction. Wiley-Liss: New York. pp 255-395. 
Devinsky W, Morrell MJ, Vogt BA (1995). Contributions of anterior cingulate cortex to behavior. Brain 118: 279-306.

Earnst KS, Kring AM (1999). Emotional responding in deficit and non-deficit schizophrenia. Psychiatry Res 88: 191-207.

First MB, Spitzer RL, Gibbon M, Williams J (1996). Structured Clinical Interview for DSM-IV Axis I disorders (SCID), Clinician Version: User's Guide. American Psychiatric Press: Washington, DC.

Fudge JL, Powers JM, Haber SN, Caine ED (1998). Considering the role of the amygdala in psychotic illness: a clinicopathological correlation. J Neuropsychiatry Clin Neurosci 10: 383-394.

Grace AA, Moore H, O'Donnell P (1998). The modulation of corticoaccumbens transmission by limbic afferents and dopamine: a model for the pathophysiology of schizophrenia. Advances in Pharmacology (New York) 42: 721-724.

Grossberg S (2000). The imbalanced brain: from normal behavior to schizophrenia. Biol Psychiatry 48: 81-98.

Gur RE, McGrath CE, Chan RM, Schroeder LF, Turner T, Turetsky BI et al (2002). An fMRI study of facial emotion processing in patients with schizophrenia. Am J Psychiatry 159: 1992-1999.

Gusnard DA, Akbudak E, Shulman GL, Raichle ME (2001). Medial prefrontal cortex and self-referential mental activity: relation to a default mode of brain function. Proc Natl Acad Sci USA 98: 4259-4264.

Haber SN, Fudge JL (1997). The primate substantia nigra and VTA: integrative circuitry and function. Crit Rev Neurobiol 11: 323-342.

Haber SN, McFarland NR (1999). The concept of the ventral striatum in nonhuman primates. Ann NY Acad Sci 877: 33-48.

Heimer L (2000). Basal forebrain in the context of schizophrenia. Brain Res Brain Res Rev 31: 205-235.

Hollingshead AB, Redlich FC (1958). Social class and mental illness. Am J Psychiatry 149: 1035-1044.

Horvitz JC (2000). Mesolimbocortical and nigrostriatal dopamine responses to salient non-reward events. Neuroscience 96 651-656.

Joyce JN, Janowsky A, Neve KA (1991). Characterization and distribution of [125I]epidepride binding to dopamine D2 receptors in basal ganglia and cortex of human brain. J Pharmacol Exp Ther 257: 1253-1263.

Kapur S (2003). Psychosis as a state of aberrant salience: a framework linking biology, phenomenology, and pharmacology in schizophrenia. Am J Psychiatry 160: 13-23.

Kring AM, Kerr SL, Smith DA, Neale JM (1993). Flat affect in schizophrenia does not reflect diminished subjective experience of emotion. J Abnorm Psychol 102: 507-517.

Kring AM, Neale JM (1996). Do schizophrenic patients show a disjunctive relationship among expressive, experiential, and psychophysiological components of emotion? J Abnorm Psychol 105: 249-257.

Lang PJ, Greenwald MK (1988). The International Affective Picture System Standardization Procedure and Initial Group Results for Affective judgments: Technical Report IA. Center for Research in Psychophysiology, University of Florida: Gainesville, FL.

Ledoux JE (1992). Emotion and the amygdala. In: Aggleton JP (ed). The Amygdala: Neurobiological Aspects of Emotion, Memory and Mental Dysfunction. Wiley-Liss: New York. pp 339-351.

Liberzon I, Phan KL, Decker LR, Taylor SF (2003). Extended amygdala and emotional salience: a PET activation study of positive and negative affect. Neuropsychopharmacology 28: 726-733.

McCarley RW, Faux SF, Shenton ME, Nestor PG, Adams J (1991). Event-related potentials in schizophrenia: their biological and clinical correlates and a new model of schizophrenic pathophysiology. Schizophr Res 4: 209-231.

Meador-Woodruff JH, Haroutunian V, Powchik P, Davidson M, Davis KL, Watson SJ (1997). Dopamine receptor transcript expression in striatum and prefrontal and occipital cortex. Arch Gen Psychiatry 54: 1089-1095.
Miller DD, Andreasen NC, O’Leary DS, Rezai K, Watkins GL, Ponto LL et al (1997). Effect of antipsychotics on regional cerebral blood flow measured with positron emission tomography [published erratum appears in Neuropsychopharmacology 1998 Apr;18(4):323-324]. Neuropsychopharmacology 17: 230-240.

Minoshima S, Koeppe RA, Frey KA, Kuhl DE (1994). Anatomic standardization: linear scaling and nonlinear warping of functional brain images. J Nucl Med 35: 1528-1537.

Minoshima S, Koeppe RA, Mintun MA, Berger K, Taylor SF, Frey KA et al (1993). Automated detection of the intercommissural (AC-PC) line for stereotactic localization of functional brain images. J Nuc Med 34: 322-329.

Mogenson GJ, Jones DL, Yim CY (1980). From motivation to action: functional interface between the limbic system and the motor system. Prog Neurobiol 14: 69-97.

Moore H, West AR, Grace AA (1999). The regulation of forebrain dopamine transmission: relevance to the pathophysiology and psychopathology of schizophrenia. Biol Psychiatry 46: 40-55.

Myin-Germeys I, Delespaul PA, deVries MW (2000). Schizophrenia patients are more emotionally active than is assumed based on their behavior. Schizophr Bull 26: 847-854.

Nauta WJH, Domesick VB (1982). Neural associations of the limbic system. In: Beckman AL (ed). The Neural Basis of Behavior. SP Medical and Scientific Books: New York. pp 175-206.

Nelson MD, Saykin AJ, Flashman LA, Riordan HJ (1998). Hippocampal volume reduction in schizophrenia as assessed by magnetic resonance imaging: a meta-analytic study. Arch Gen Psychiatry 55: 433-440.

Nuechterlein KH, Dawson ME (1984). Information processing and attentional functioning in the developmental course of schizophrenic disorders. Schizophr Bull 10: 160-203.

Ochsner KN, Bunge SA, Gross JJ, Gabrieli JD (2002). Rethinking feelings: an FMRI study of the cognitive regulation of emotion. J Cogn Neurosci 14: 1215-1229.

Overall JE, Gorham DR (1962). Brief psychiatric rating scale. Psychol Rep 10: 799-812.

Paradiso S, Andreasen NC, Crespo-Facorro B, O’Leary DS, Watkins GL, Boles Ponto LL et al (2003). Emotions in unmedicated patients with schizophrenia during evaluation with positron emission tomography. Am J Psychiatry 160: 1775-1783.

Parkinson JA, Cardinal RN, Everitt BJ (2000). Limbic corticalventral striatal systems underlying appetitive conditioning. Prog Brain Res 126: 263-285.

Pearlson GD, Barta PE, Powers RE, Menon RR, Richards SS, Aylward EH et al (1997). Medial and superior temporal gyral volumes and cerebral asymmetry in schizophrenia versus bipolar disorder. Biol Psychiatry 41: 1-14.

Pessoa L, McKenna M, Gutierrez E, Ungerleider LG (2002). Neural processing of emotional faces requires attention. Proc Natl Acad Sci USA 99: 11458-11463.

Pfefferbaum A, Ford JM, White PM, Roth WT (1989). P3 in schizophrenia is affected by stimulus modality, response requirements, medication status and negative symptoms. Arch Gen Psychiatry 46: 1035-1044.

Phan KL, Wager T, Taylor SF, Liberzon I (2002). Functional neuroanatomy of emotion: a meta-analysis of emotion activation studies in PET and fMRI. Neuroimage 16: 331-348.

Phillips ML, David AS (1994). Understanding the symptoms of schizophrenia using visual scan paths. $B r J$ Psychiatry 165: 673-675.

Phillips ML, David AS (1998). Abnormal visual scan paths: a psychophysiological marker of delusions in schizophrenia. Schizophr Res 29: 235-245.

Phillips ML, Williams L, Senior C, Bullmore ET, Brammer MJ, Andrew C et al (1999). A differential neural response to threatening and non-threatening negative facial expressions in paranoid and non-paranoid schizophrenics. Psychiatry Res 92: $11-31$. 
Renshaw PF, Yurgelun-Todd DA, Cohen BM (1994). Greater hemodynamic response to photic stimulation in schizophrenic patients: an echo planar MRI study. Am J Psychiatry 151: 1493-1495.

Reynolds SM, Berridge KC (2002). Positive and negative motivation in nucleus accumbens shell: bivalent rostrocaudal gradients for GABA-elicited eating, taste 'liking'/'disliking' reactions, place preference/avoidance, and fear. J Neurosci 22: 7308-7320.

Reynolds SM, Berridge KC (2003). Glutamate motivational ensembles in nucleus accumbens: rostrocaudal shell gradients of fear and feeding. Eur J Neurosci 17: 2187-2200.

Rolls ET (1996). The orbitofrontal cortex. Philos Trans $R$ Soc London B Biol Sci 351: 1433-1443; discussion 1443-1444.

Saykin AJ, Gur RC, Gur RE, Mozley PD, Mozley LH, Resnick SM et al (1991). Neuropsychological function in schizophrenia. Arch Gen Psychiatry 48: 618-624.

Schneider F, Weiss U, Kessler C, Salloum JB, Posse S, Grodd W et al (1998). Differential amygdala activation in schizophrenia during sadness. Schizophr Res 34: 133-142.

Seeman P (1987). Dopamine receptors and the dopamine hypothesis of schizophrenia. Synapse 1: 133-152.

Siegel BV, Buchsbaum MS, Bunney WE, Gottschalk LA, Haier RJ, Lohr JB et al (1993). Cortical-strial-thalamic circuits and brain glucose metablic activity in 70 unmedicate male schizophrenic patients. Am J Psychiatry 150: 1325-1336.

Stern CE, Passingham RE (1996). The nucleus accumbens in monkeys (Macaca fascicularis): II. Emotion and motivation. Behav Brain Res 75: 179-193.
Streit M, Wolwer W, Gaebel W (1997). Facial-affect recognition and visual scanning behaviour in the course of schizophrenia. Schizophr Res 24: 311-317.

Takahashi H, Koeda M, Oda K, Matsuda T, Matsushima E, Matsuura $M$ et al (2004). An fMRI study of differential neural response to affective pictures in schizophrenia. Neuroimage 22: 1247-1254.

Taylor SF, Liberzon I, Decker LR, Koeppe RA (2002). A functional anatomic study of emotional experience in schizophrenia. Schizophr Res 58: 159-172.

Taylor SF, Liberzon I, Koeppe RA (2000). The effect of graded aversive stimuli on limbic and visual activation. Neuropsychologia 38: 1415-1425.

Taylor SF, Phan KL, Decker LR, Liberzon I (2003). Subjective rating of emotionally salient stimuli modulates neural activity. Neuroimage 18: 650-659.

Taylor SF, Tandon R, Koeppe RA (1997). PET study of greater visual activation in schizophrenia. Am J Psychiatry 154: 1296-1298.

Williams LM, Das P, Harris AW, Liddel BB, Brammer MJ, Olivieri G et al (2004). Dysregulation of arousal and amygdala-prefrontal systems in paranoid schizophrenia. Am J Psychiatry 161: 480-489.

Wolkin A, Sanfilipo M, Duncan E, Angrist B, Wolf AP, Cooper TB et al (1996). Blunted change in cerebral glucose utilization after haloperidol treatment in schizophrenic patients with prominent negative symptoms. Am J Psychiatry 153: 346-354.

Wright IC, Rabe-Hesketh S, Woodruff PW, David AS, Murray RM, Bullmore ET (2000). Meta-analysis of regional brain volumes in schizophrenia. Am J Psychiatry 157: 16-25. 